\title{
Device simulation and fabrication of field effect solar cells
}

\author{
KAORI MIYAZAKI*, NOBUYUKI MATSUKI, HIROYUKI SHINNO, HIROSHI FUJIOKA ${ }^{\dagger}$, \\ MASAHARU OSHIMA ${ }^{\dagger}$ and HIDEOMI KOINUMA \\ Ceramic Materials and Structures Laboratory, Tokyo Institute of Technology, 4259 Nagatsuta-cho, Midori-ku, \\ Yokohama-shi, Kanagawa, 226-8503, Japan \\ 'Department of Applied Chemistry, The University of Tokyo, 7-3-1 Hongou Bunkyo-ku, Tokyo, 113-8656, Japan
}

\begin{abstract}
The performance of a novel hydrogenated amorphous silicon $(a-\mathrm{Si}: \mathrm{H})$ solar cell which utilizes the field effect solar cell (FESC) has been investigated both theoretically and experimentally. The theoretical analysis has been done for both $\boldsymbol{p}$ - and $\boldsymbol{n}$-channel FESCs by employing a two-dimensional device simulator which is based on current continuity and Poisson equations. The calculated performance is compared with that of conventional $(p-i-n) a-S i: H$ solar cells. The calculation demonstrated that both $n$-channel and $p$-channel FESCs could improve the conversion efficiency by as much as $50 \%$.

In order to check the reliability of simulation, the transport properties of intrinsic $a$-Si : $\mathrm{H}$ film and thin film transistor (TFT) have also been calculated and compared with the experimentally obtained characteristics. Experimental verification of TFT and FESC has been attempted by using $\mathrm{MgO}$ and $a$-SiN : $\mathrm{H}$ as dielectric layer materials. Preliminary results are presented.
\end{abstract}

Keywords. Two-dimensional device simulation; field effect; $a$-Si : $\mathrm{H}$; solar cell.

\section{Introduction}

Despite its high possibility of large scale and low cost production, hydrogenated amorphous silicon $(a-\mathrm{Si}: \mathrm{H})$ solar cells still suffer from low conversion efficiency and photo instability. A main reason for the low efficiency is the high localized state density in the high-dope window layer.

Recently, we proposed a new type of $a-\mathrm{Si}: \mathrm{H}$ solar cell in which $p$-type layer is designed to be induced by the field effect (FE) instead of the impurity doping (Koinuma et al 1996). This new structure is expected to eliminate the window layer problem mentioned above. In addition, we can use any metal, instead of a transparent conductive oxide, as top contact (comb) electrode, since it covers only a small portion of the cell. Thus, we have a freedom of choosing a metal with proper work function. A two-dimensional device simulation supported our concept; the collection efficiency for short wavelength light was calculated to increase remarkably in the FESC. Improvements in the open circuit voltage $\left(V_{O C}\right)$ and the fill factor $(F F)$ were also predicted by the use of comb electrodes with large work functions (Fujioka et al 1998). In these calculations, we employed p-channel FESCs in view of the fact that $p-i-n$ structures have been exclusively used in conventional cells. In this paper, we deploy our device simulation to $n$-channel FESC and thin film transistor as well.

\footnotetext{
*Author for correspondence
}

\section{Device simulation}

Since the FESC involves carrier motion in both lateral and vertical directions to the surface, it is essential to analyze the cell characteristics using a two-dimensional simulator. We employed SILVACO's ATLAS for twodimensional device simulation using the current continuity and the Poisson equations. Different from the frequently used assumption that the temperature is $0 \mathrm{~K}$ to determine the ionized trap concentration, we used accurate ShockleyRead-Hall (Hall 1952) expression at $300 \mathrm{~K}$ to obtain both the trap occupation function and recombination rate.

The solar cell performances of dielectric/a-Si: $\mathrm{H} i-n /$ metal ( $p$-channel FE) and dielectric/a-Si : $\mathrm{H}$ i-p/metal (n-channel FE) cells (figure 1) are calculated and compared with those of conventional $p-i-n$, i.e. ITO/ $a-\mathrm{Si}: \mathrm{H} p-i-n /$ metal and $n-i-p$, i.e. ITO/a-Si : $\mathrm{H} n-i-p /$ metal cells. The width of the comb front contact is assumed to be $1 \mu \mathrm{m}$. The electric field across the insulator to form the surface inversion layer can be achieved either by the use of fixed charge in the insulator (Hezel and Schörner 1981) or the application of bias to a dielectric or ferroelectric material. In our simulation, we chose the bias application to MOS (metal-dielectric oxide- $a-\mathrm{Si}: \mathrm{H}$ semiconductor) structure for simplicity.

For simulation of such a defective material as $a-\mathrm{Si}: \mathrm{H}$, it is important to model a deep level in the energy gap appropriately. We assumed the exponential tail and Gaussian localized distribution (Shaw and Hack 1988). The donor-like states become positive when they ionize, while the acceptor-like states become negative. No 
additional trap is assumed at the insulator $/ a-\mathrm{Si}: \mathrm{H}$ interface because our cell is not sensitive to the interface trap due to the high electric field near the interface (Kuwano et al 1981).

For performing the device simulation with this structure, there are many material and structural parameters as shown in figure 1, which include density of defect states $\left(N_{\mathrm{D}}\right.$ and $\left.N_{\mathrm{G}}\right)$, capture cross section, mobility $\left(\mu_{\mathrm{c}}\right.$ and $\left.\mu_{\mathrm{h}}\right)$, refractive index ( $n$ and $k$ ), dielectric constant $\left(\varepsilon_{\mathrm{r}}\right)$, work function $(\Phi)$, surface velocity, mobility gap $\left(E_{\mathrm{g}}\right)$, doping concentration, FE-induced charge density, film thickness $(d)$, and spacing of the comb front contacts $(l)$. In this

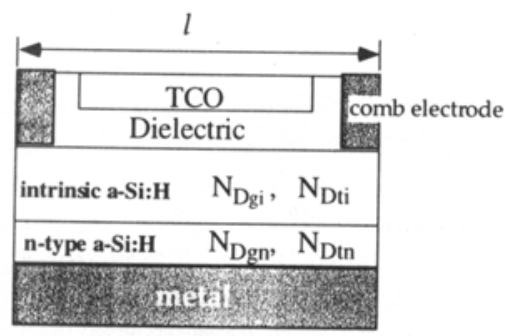

(a)

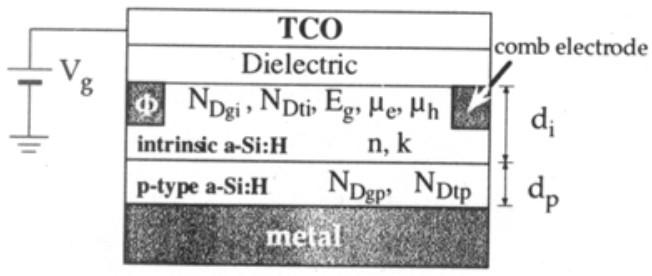

(b)

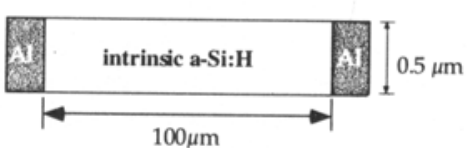

(c)

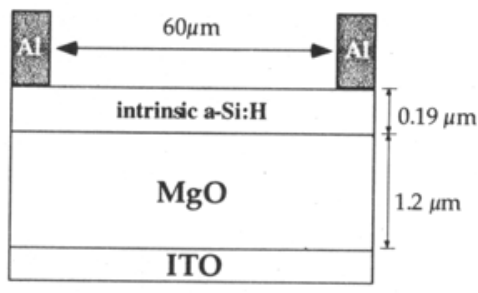

(d)

Figure 1. Schematic cross-sections of $p$-channel (a) and $n$-channel (b) FESCs, a sample to calculate conductivity of $i-a-\mathrm{Si}: \mathrm{H}(\mathbf{c})$, and an $a-\mathrm{Si}: \mathrm{H}$ TFT (d).

Table 1. Parameters for simulation.

\begin{tabular}{|c|c|}
\hline Mobility gap & $1.7 \mathrm{eV}$ \\
\hline Mobility (electron) & $25 \mathrm{~cm}^{2} \mathrm{~V}^{-1} \mathrm{sec}^{-1}$ \\
\hline Mobility (hole) & $6 \mathrm{~cm}^{2} \mathrm{~V}^{-1} \mathrm{sec}^{-1}$ \\
\hline Charge capture cross-section & $1 \times 10^{-14} \mathrm{~cm}^{2}$ \\
\hline Neutral capture cross-section & $1 \times 10^{-16} \mathrm{~cm}^{2}$ \\
\hline Donor tail energy & $46 \mathrm{meV}$ \\
\hline Acceptor tail energy & $27 \mathrm{meV}$ \\
\hline Gap state density in $i$-layer & $1.5 \times 10^{15} \mathrm{~cm}^{-3} \cdot \mathrm{eV}^{-1}$ \\
\hline Gap state density in doping layer & $6.0 \times 10^{18} \mathrm{~cm}^{-3} \cdot \mathrm{eV}^{-1}$ \\
\hline $\begin{array}{l}\text { Effective density of states at } \\
\text { conduction/valence band edge }\end{array}$ & $2 \times 10^{20} \mathrm{~cm}^{-3} / 2 \times 10^{20} \mathrm{~cm}^{-3}$ \\
\hline Thickness of $p$-type $/ n$-type layer & $200 \AA / 200 \AA$ \\
\hline Doping concentration in $p$-type layer & $6 \times 10^{18} \mathrm{~cm}^{-3}$ \\
\hline Doping concentration in $n$-type layer & $6 \times 10^{18} \mathrm{~cm}^{-3}$ \\
\hline Photon flux & $\mathrm{AM}-1.5100 \mathrm{~mW} \cdot \mathrm{cm}^{-2}$ \\
\hline Reflectance at the front side of $a-\mathrm{Si}: \mathrm{H}$ layer & 0.2 \\
\hline Reflectance at the back side of $a-\mathrm{Si}: \mathrm{H}$ layer & 0.8 \\
\hline Absorptance of TCO layer for conventional cells & $0 \cdot 1$ \\
\hline Absorptance of dielectric layer for FESCs & 0.0 \\
\hline Absorption coefficient of $a-\mathrm{Si}: \mathrm{H}$ & Literature values (Piller 1985) \\
\hline Induced charge density at the interface & $5 \times 10^{12}$ and $1 \times 10^{13} \mathrm{~cm}^{-2}$ \\
\hline$i$-layer thickness & $0.3 \sim 0.7 \mu \mathrm{m}$ \\
\hline Comb electrode spacing & $15 \mu \mathrm{m} \sim 200 \mu \mathrm{m}$ \\
\hline
\end{tabular}


study, we focus on the effects of intrinsic $(i)$ layer thickness and the distance between comb-electrodes. The $i$-layer thickness ranges from $3000 \AA$ to $7000 \AA$. The comb-electrode distance is varied from $15 \mu \mathrm{m}$ to $200 \mu \mathrm{m}$. The FE-induced charge is fixed at $5 \times 10^{12}$ or $1 \times 10^{13} \mathrm{~cm}^{-2}$. The light intensity is $A M-1.5,100 \mathrm{~mW} / \mathrm{cm}^{2}$. The parameters used in the calculation are listed in table 1.

\section{Experimental}

To check the validity of the simulation, a thin film transistor (TFT) using $\mathrm{MgO}$ dielectric layer was fabricated and their electric property was compared with the calculation. Structure of the TFT is shown in figure 1d. First, a $1.2 \mu \mathrm{m}$ thick $\mathrm{MgO}$ dielectric layer was deposited on an ITO/glass substrate by pulsed laser deposition (PLD) at a substrate temperature of $350^{\circ} \mathrm{C}$. Then, a $1900 \AA$ thick intrinsic $a-\mathrm{Si}: \mathrm{H}$ was deposited by plasma CVD. Finally, aluminum source and drain electrodes were deposited $(\mathrm{L} / \mathrm{W}=60 \mu \mathrm{m} / 2.7 \mathrm{~mm})$ on the $a-\mathrm{Si}: \mathrm{H}$ by the vacuum evaporation.

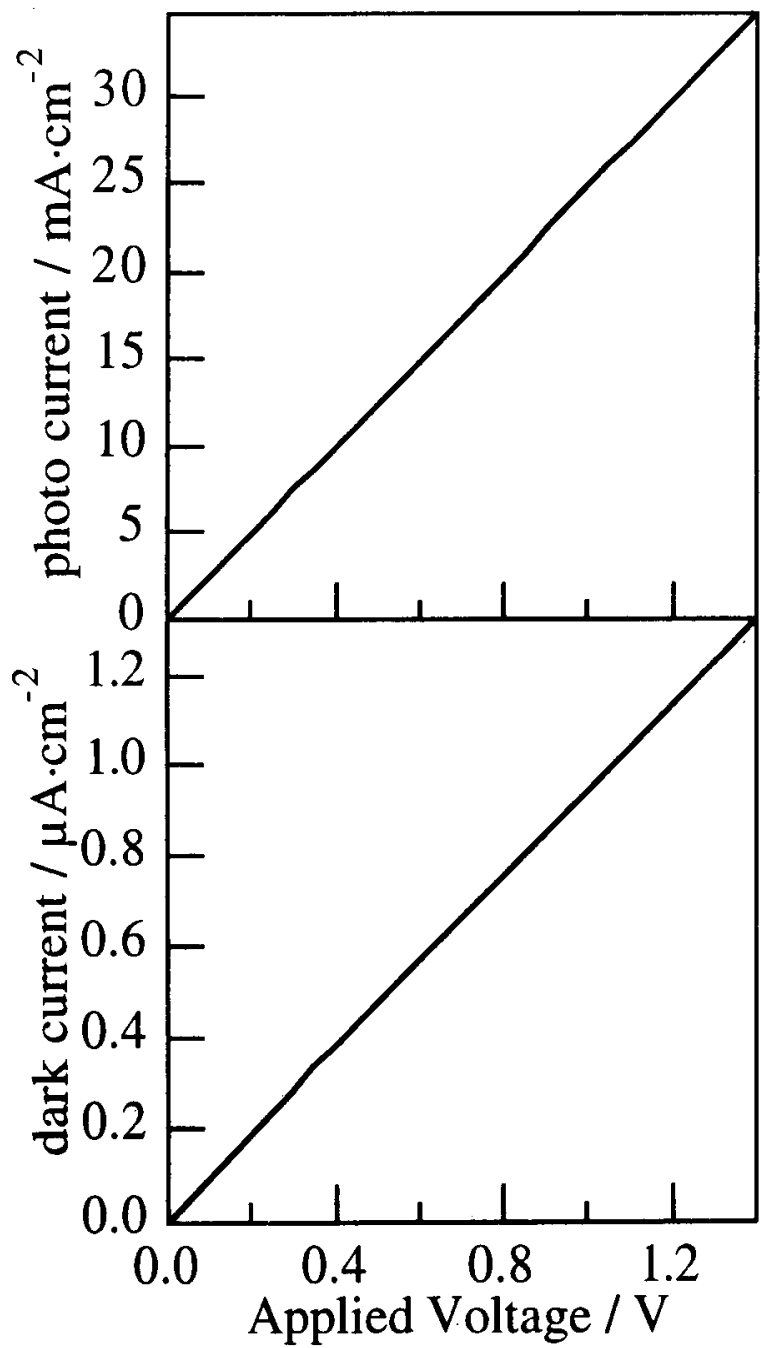

Figure 2. $I-V$ characteristic of $i-a-\mathrm{Si}: \mathrm{H}$ under the light irradiation and dark condition.

\section{Results}

\subsection{Characteristics of intrinsic a-Si: $H$}

In order to validate the parameters for $a-S i: H$, the conductivities of intrinsic $a-\mathrm{Si}: \mathrm{H}(i-a-\mathrm{Si}: \mathrm{H})$ have been calculated by the same simulator. The schematic crosssection of the structure used to calculate conductivities is shown in figure 1c. Photo and dark currents of $i-a-\mathrm{Si}: \mathrm{H}$ are calculated under $\mathrm{AM}-1.5100 \mathrm{~mW} / \mathrm{cm}^{2}$ and $1 \times 10^{-15} \mathrm{~mW} / \mathrm{cm}^{2}$ light irradiation, respectively. Figure 2 shows the $I-V$ characteristics of $i-a-\mathrm{Si}: \mathrm{H}$ under light and dark conditions. Photo and dark conductivities are evaluated to be $2 \times 10^{-4} \mathrm{~S} / \mathrm{cm}$ and $9 \times 10^{-9} \mathrm{~S} / \mathrm{cm}$, respectively, which are consistent with observed values.

\subsection{Thin film transistor}

$V_{\mathrm{G}}-I_{\mathrm{D}}$ characteristics calculated by taking the dielectricl semiconductor interface defects into account agreed well with the best reported data (Tsukada 1991). Figure 3 shows the $V_{\mathrm{D}}-I_{\mathrm{D}}$ characteristics we obtained in our $a-\mathrm{Si}: \mathrm{H} / \mathrm{MgO} / \mathrm{TO}$ structure. However, it was not so good probably due to the leakage and/or insufficient resistivity in $\mathrm{MgO}$ layer. The calculated on/off ratio varies from $10^{5}$ to $10^{10}$ with the decrease in the interface defect density from $5 \times 10^{11} \mathrm{~cm}^{-2} \cdot \mathrm{eV}^{-1}$ to zero, while that of our TFT is $10^{3}$.

\subsection{Field effect solar cell}

The typical $i$-layer thickness and the spacings of the comb front contacts both for $n$ - and $p$-channel cells

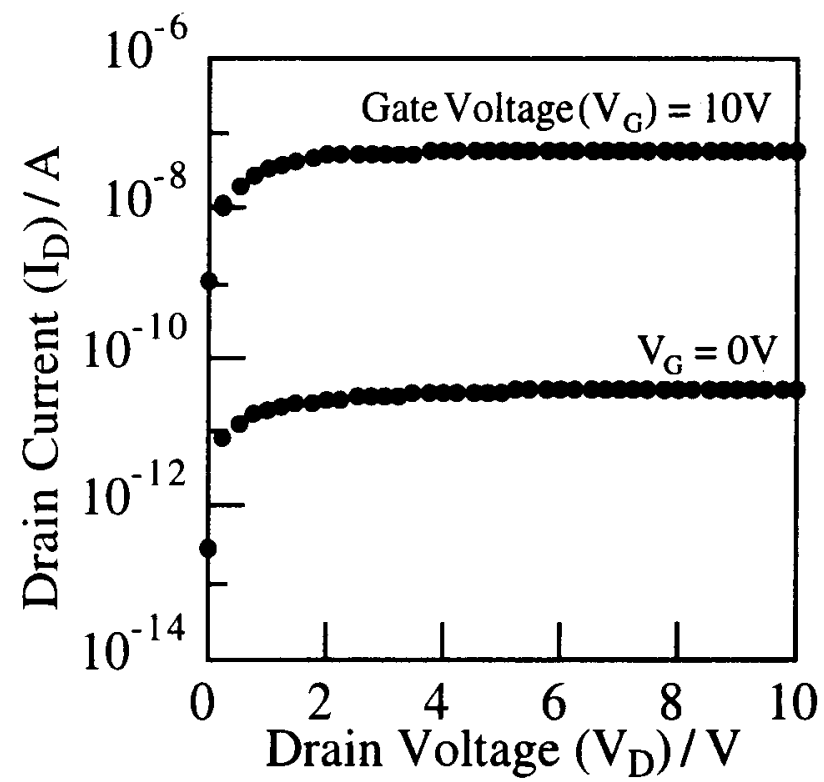

Figure 3. Experimental $I_{\mathrm{D}}-V_{\mathrm{D}}$ characteristics of an $a-\mathrm{Si}: \mathrm{H}$ TFT. 
(figures $1 \mathrm{a}, \mathrm{b}$ ) are $0.5 \mu \mathrm{m}$ and $30 \mu \mathrm{m}$, respectively. In the dark, the energy band diagrams along the interface between the dielectric and $a-\mathrm{Si}: \mathrm{H}$ are flat. However, light irradiation causes a band bending. The band profile along the interface of the $n$-channel FESC becomes concave while that of the p-channel FESC becomes convex. The concaved band allows electrons at the interface to move to the comb-electrodes and the convex band diagram allows holes to move to the combelectrodes. Therefore, electrons of the $n$-channel FESC whose majority carrier at the interface is electron, can reach the comb-electrodes, and holes of the p-channel FESC whose majority carrier at the interface is hole, can reach the comb-electrodes.

The possible maximum output powers of various types of $a-\mathrm{Si}: \mathrm{H}$ solar cells are calculated as a function of $i$-layer thickness. The $n$ - and $p$-channel FESCs with a comb front contact spacing of $30 \mu \mathrm{m}$ give powers higher than the conventional $p-i-n$ cell. The $i$-layer thickness to give the maximum output power for the $n$-channel FESC and $n-i-p$ junction are about $0.4 \mu \mathrm{m}$, which are thinner than those $(0.6 \mu \mathrm{m})$ for the $p$-channel FESC and $p-i-n$ junction cell.

Figure 4 shows the possible maximum output powers of $a-\mathrm{Si}: \mathrm{H}$ solar cells calculated as a function of the comb electrode spacing. The $n$-channel FESC shows smaller spacing dependence of the maximum output power. This result should be noted as an advantage of $n$-channel cell in view of the generally accepted notion that $p-i-n$ cell is better than $n-i-p$ cell.

\section{Discussion}

The maximum output powers for both $n$ - and $p$-channel FESCs are larger than those for conventional cells. This can be presumed to come from the fact that the FESCs use window layers induced by the field effect instead of the impurity doped layers and that the FESCs can take any metals as a top contact electrode instead of TCO at the cost of effective area. The doping free window layer brings the improvement in short-circuit current $\left(I_{\mathrm{SC}}\right)$ due to the increased quantum efficiency for light with short wavelength. The work function of the comb electrode changes the barrier height, which directly influence $V_{O C}$ and $F F$. For the $n$-channel FESCs, the smaller work functions of comb-electrodes lead to smaller Schottky barriers. Hence, we can improve the $V_{\text {oc }}$ and $F F$ by the use of a comb electrode with a small work function.

The maximum output powers for $n$-channel FESCs with comb electrode spacings $<50 \mu \mathrm{m}$ are slightly smaller than those for $p$-channel FESCs. This can be attributed to the difference in the type of the carriers which drift in the direction normal to the surface. It is well known that the mobility of hole in $a-\mathrm{Si}: \mathrm{H}$ is much smaller than that of electron. Since a large portion of carriers in the $a-\mathrm{Si}: \mathrm{H}$ are generated near the surface, holes in the $n$-channel FESCs must travel long distance, which in turn results in the higher possibility of recombination with the electrons. This difference in the type of carriers gives the result that the optimum $i$-layer thicknesses give the maximum output power for the $n$-channel FESC and $n-i-p$ junction are slightly smaller than those for the $p$-channel FESC and $p-i-n$ junction. The short hole drift distance is essentially important for the $n$ channel FESCs, while the large volume in which the hole-electron pairs are generated is important for the $p$-channel FESCs.

On the other hand, $n$-channel FESCs with long comb electrode spacing show higher efficiency when they are compared with $p$-channel FESCs with the same spacings. For the FESCs with large comb electrode spacing, the lateral motion of the carriers limits the cell performance. In the $n$-channel cells, we can take advantage of high mobility of the electrons in this lateral transportation. The use of $n$-channel cells allows us to use low cost printing technique for the production of FESCs, which makes the $n$-channel cells attractive for mass-production.

\section{Conclusion}

Our simulation has shown that both $n$ - and $p$-channel $a$-Si : H FESCs offer better performance than conventional cells. Furthermore, $n$-channel FESCs with comb electrode

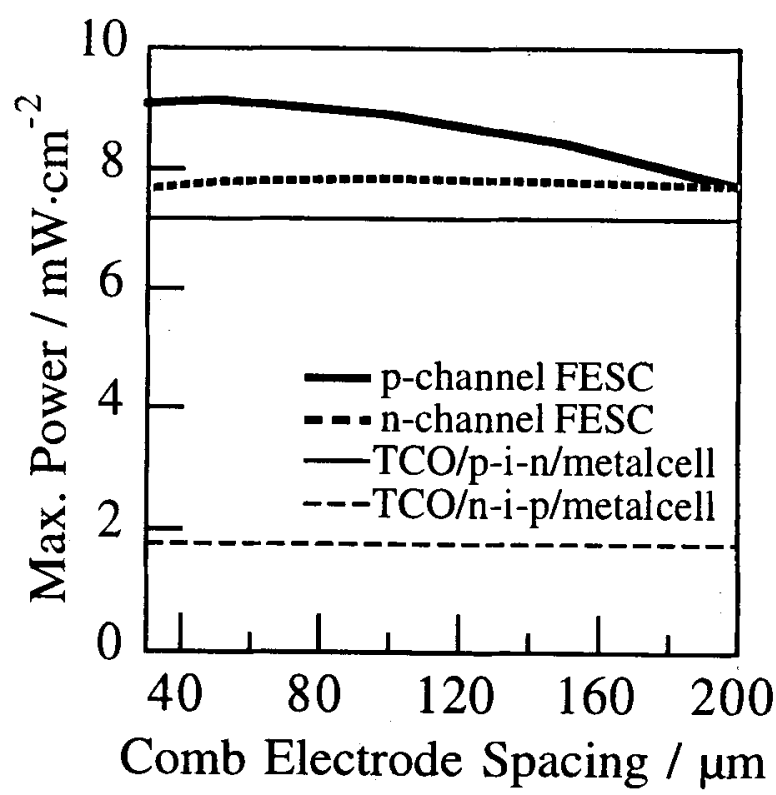

Figure 4. Maximum output power profiles calculated as a function of the comb front electrode spacing. The $i$-layer thickness and FE-induced charge density were assumed to be $0.4 \mu \mathrm{m}$ for $n$-channel FESC and $n-i-p$ junction, $0.6 \mu \mathrm{m}$ for $p$-channel FESC and $p-i-n$ junction, and $1 \times 10^{13} \mathrm{~cm}^{-2}$. 
spacing as wide as $100 \mu \mathrm{m}$ can give conversion efficiencies maintained at levels higher than conventional $p-i-n$ cell. The electric characteristics of the $a-\mathrm{Si}: \mathrm{H}$ TFT calculated by taking the dielectric/semiconductor interface defects agree well with the best reported data, but our preliminary result was not so good. Further studies are in progress to verify the possibility of FESC.

\section{Acknowledgements}

Authors wish to thank SILVACO Inc. for its support in device simulation. This work was supported by NEDO International Joint Research Grant.

\section{References}

Fujioka H, Oshima M, Hu C, Matsuki N, Miyazaki $\mathrm{K}$ and Koinuma H $1998 \mathrm{~J}$. Non-Cryst. Solids 227-230 1287

Hall R N 1952 Phys. Rev. 87387

Hezel R and Schörner R 1981 J. Appl. Phys. 523076

Koinuma H, Fujioka H, Hu C, Koida T and Kawasaki M 1996 Mater. Res. Soc. Symp. Proc. 42695

Kuwano Y, Fukatsu T, Imai T, Ohnishi M, Nishiwaki $\mathrm{H}$ and Tsuda S 1981 Jpn J. Appl. Phys. supp. 2020

Piller H 1985 Handbook of optical constants of solids (Academic Press Inc.)

Shaw T and Hack M 1988 J. Appl. Phys. 6419457

Tsukada T 1991 Digest 49th annual device research conf. (Colorado: IEEE Electron Devices Society) IA-3 